\title{
Ensino de estatística com enfoque CTS: uma articulação entre matemática e temas sociais
}

\section{Teaching statistics with focus CTS: a linking mathematics and social issues}

Luciana Boemer Cesar Pereira ${ }^{1}$ | lucesarboemerpg@yahoo.com.br

Rosemari Monteiro Castilho Foggiatto Silveira ${ }^{1}$

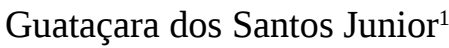

\section{RESUMO}

Este artigo apresenta resultados da realização de uma proposta de ensino de conceitos estatísticos numa abordagem CTS. O estudo buscou apresentar aos estudantes a articulação da disciplina de matemática a partir de temas sociais da sua realidade. Quanto a natureza a pesquisa se caracteriza como aplicada, abordagem metodológica foi a qualitativa e de cunho interpretativo, foi realizada na disciplina de matemática, em um $6^{\circ}$ ano do ensino fundamental de uma escola do campo e atendeu a um dos objetivos dos documentos curriculares da disciplina de matemática, que é o trabalho com informações estatísticas e representações de dados. As atividades realizadas contribuíram para a aprendizagem de técnicas de organização de dados estatísticos e proporcionou reflexões destes a partir da realidade dos discentes como propõe os documentos oficiais e formar alunos reflexivos em condições de discutir a realidade que os cerca.

Palavras-chave: estatística, CTS, Escola do campo, Cultura de tabaco.

\section{ABSTRACT}

This article presents results of a proposal for teaching statistical concepts which was carried out in an STS approach. The study aimed at presenting the students the articulation of the Math course, taking social themes of their reality as a starting point. Regarding the nature of the research, which is characterized as applied research, the methodological approach was the qualitative one, with an interpretative characteristic. It was carried out in the Math course, in the sixth grade of an elementary school in the countryside, and it fulfilled one of the aims of the curricular documents of Math, which is working with statistical information and data representations. The activities accomplished contributed to the learning of techniques of organizations of statistical data and also gave rise to reflections with basis on these data inferring the students' reality, as proposed by official documents, to form reflexive thinking students in condition to discuss the reality around them.

Key Words: statistics, STS, school in the countryside, tobacco growing.

1 Universidade Tecnológica Federal do Paraná (UTFPR) 


\section{INTRODUÇÃO}

A cultura de tabacos tem se alastrado pela região sul nos últimos tempos, classificando o Brasil como um dos maiores produtores do mundo. Essa cultura é praticada em regime de cultivo familiar onde o trabalho é intensivo e há uma enorme utilização de agrotóxicos e de lenhas consumida nas estufas de secagem.

Glantz et al. (1996), descreve que a saúde das famílias dos agricultores que cultivam o fumo são agredidos de diversas formas, tanto pelo uso de agrotóxicos quanto pelo contato com a planta úmida, que libera nicotina e ainda, também pelo cheiro das folhas durante a secagem nas estufas.

Um levantamento realizado com fumicultores na região Sul do Brasil concluiu que 55\% deles não usam roupas de proteção, tais como máscaras, luvas e botas. Eles justificam essa situação com argumentos sobre os altos custos dos equipamentos e sua inadequação ao clima tropical. A pesquisa também concluiu que cerca de $50 \%$ dos familiares dos agricultores sofrem de problemas de saúde associados ao uso de substâncias químicas, como dores de cabeça persistentes e vômitos, e $42 \%$ conhecem alguém com problemas físicos de nascença (BRASIL, 2008).

As estatísticas e pesquisas apontam que o agricultor que trabalha nesta cultura está exposto a inúmeros riscos a sua saúde. Além disso, há grandes e poderosas corporações transnacionais que industrializam o fumo e conseguem, maquiando e camuflando, explorar os agricultores e suas famílias.

As fumageiras, hoje, não precisam obrigar o produtor a absorver insumos, optar por seguro ou construções de investimento, pois o fumicultor já está irremediavelmente atrelado ao esquema e seus instrutores controlam a fidelidade. Os fumicultores reconhecem que estão intoxicados e com suas vidas e a de seus filhos seriamente comprometidas. Também não acreditam que seja possível produzir sem esta relação opressiva, e nem sequer acreditam que existam alternativas. Submetem-se. $E$ a ideologia do dominador passa a ser a visão do dominado. Infelizmente, este é o auge da servidão (PINHEIRO e LUZ, 1998, p.155).

Este é um apontamento do ano 1998, mas ainda é refletida nos dias de atuais. A abordagem CTS (Ciência, Tecnologia e Sociedade), enfoca situações como esta, ou seja, é uma área de estudos que se baseia nas relações de causas, consequências e soluções para os problemas sociais existentes entre a ciência e a tecnologia. Os estudos CTS buscam a reflexão sobre os fenômenos sociais e as condições da existência humana sob a perspectiva da ciência e da tecnologia. (ANELE, 2007; VON LINSINGEN, 2007; BAZZO, 2002).

O cultivo de tabaco requer uso de grande quantidade de agrotóxicos, prejudicando a saúde dos fumicultores e o meio ambiente.

O uso de agrotóxicos é considerado inovador na agricultura, mas, seus impactos no meio ambiente e na saúde dos sujeitos que fazem as aplicações, são erros muitas vezes, irreversíveis. Produtores rurais que fazem uso descontroladamente de agrotóxicos tornam-se corresponsáveis em provocarem danos eminentes à própria saúde, além da contaminação dos alimentos e do solo, afetando o meio ambiente e a sociedade na qual ele está embutido. (ALBUQUERQUE, 2000).

Diante do exposto, este artigo tem como objetivo apresentar resultados da realização de uma proposta de ensino de conceitos estatísticos numa abordagem CTS. 


\section{O ENSINO DE ESTATÍSTICA EM BUSCA DE UM DESENVOLVIMENTO SUSTENTÁVEL NO CAMPO}

O Ensino de Estatística é responsável por desenvolver nos alunos as habilidades de coletar dados, organizar e interpretar, bem como fazer inferências. A inferência estatística é promotora do desenvolvimento e da capacidade de inferir críticas e análises. Logo, para que haja um estudo de Estatística é necessário aperfeiçoar o pensamento lógico e o raciocínio formal, entendendo primeiramente o mundo em sua volta. Batanero (2000) aponta que o grau de desenvolvimento de um país, está ligado ao modo de se colocar, de forma estatística, as situações que regem o país, de tal forma que esses dados possam ser utilizados para nortear as tomadas de decisões em diversas áreas sociais.

O mundo real, no qual o aluno está inserido tem significados e é um meio de aprendizagem para ele. Sendo assim é dever da escola proporcionar ao estudante, desde a educação básica inicial, a concepção de conceitos e definições que possam auxiliá-lo em sua pratica cidadã (LOPES, 2008).

O Ensino de Estatística em uma escola do campo tem um importante papel. A Educação no campo é responsável pelo desenvolvimento das comunidades onde as escolas se situam, pois é através de sua ação e construção educativa que as comunidades escolares do campo buscam uma maior integração social, cultural e econômica além de ser um veículo difusor de conhecimento e saberes sociais (ZANON \& WIZNIEWSKY, 2011). Neste contexto, a escola deve assumir um papel de integrar as trocas dos saberes e técnicas que apontem para estratégias de um desenvolvimento agrário sustentável.

A expressão desenvolvimento sustentável é uma perspectiva econômica com o intuito de "internalizar as externalidades ambientais, para valorizar a natureza, recodificando a ordem da vida e da cultura em termos de um capital natural e humano” (LEFF, 2004, p. 247). A educação ambiental e socioambiental são visões que tendem a incorporar uma visão crítica das relações homem e natureza.

Contudo, essa questão aponta possibilidades de uma integração numa perspectiva de assumir críticas ao analisar dados de questões socioambientais. Os dados estatísticos são bombardeados a todo o momento para a população e são responsáveis pelas tomadas de decisões das políticas sociais. São a partir de dados estatísticos alarmantes de desmatamentos, epidemias, impactos e outros fenômenos que se inserem indagações, e políticas de mudança e projetos de uma busca pela sustentabilidade começam a ser elaborados e colocados em prática.

\section{METODOLOGIA}

O estudo quanto à natureza se classifica como aplicada, abordagem metodológica foi a qualitativa de natureza interpretativa com observação participante. Para a coleta de dados utilizou-se como técnicas: anotações em diário de campo, observação, produção dos alunos e fotos. O enfoque CTS foi utilizado na forma de enxerto como proposto por Bazzo (1998), Silveira (2007).

O estudo foi desenvolvido com 31 alunos de uma escola do campo do município de Imbituva - Paraná, dos quais, aproximadamente $90 \%$ são filhos de fumicultores.

O trabalho foi realizado na disciplina de matemática, cujo objetivo era trabalhar com informações estatísticas. Para tanto, como os alunos são filhos de fumicultores foram utilizados dados de diversas fontes de pesquisa em revistas científicas e sites da SEAB (Secretaria da Agricultura e Abastecimento), DERAL (Departamento de Economia Rural) e AFULBRA (Associação de Fumicultores do Brasil) sobre a cultura de tabacos, a fim de promover reflexões sobre as implicações sociais dessa cultura. 
Para a realização do estudo foram utilizadas 4h/aulas de matemática no mês de setembro de 2012 e contou com três etapas:

1. Estudo sobre o tema;

2. Utilização dos dados estatísticos na elaboração dos gráficos e tabelas;

3. Preparação do material para a apresentação à comunidade.

Os temas foram trabalhados no conjunto com a leitura de 3 (três) textos:

- Texto 1 - Análise da conjuntura agropecuária (SEAB/DERAL) - Fumicultura - Safra 2011/12 (GROXKO, 2011, p. 1-12)

- Texto 2 - Agrotóxicos e o risco à saúde entre fumicultores. (ALMEIDA et al., 2011, p. 57-63 )

- Texto 3 - Impacto da restrição ao uso de aditivos em derivados deotabaco nos principais municípios fumicultores do Paraná. (ZANCHET \& LIMA, 2012, p.58-71)

Os conteúdos do currículo de matemática foram articulados às informações que estes traziam. Após o trabalho de leitura, foram realizadas as construções de gráficos e tabelas e preparado em forma de banners para apresentar à comunidade.

Os temas trabalhados foram:

Produção Brasileira de tabacos;

Evolução da cultura de tabacos;

Produção Regional de tabacos;

Impactos na saúde dos fumicultores;

Esta é uma pesquisa que se caracteriza numa perspectiva pedagógica, configurada como "uma ação que cientificiza a prática educativa, a partir de princípios éticos que visualizam a contínua formação e emancipação de todos os sujeitos da prática” (FRANCO, 2005, p. 483). A análise dos dados foi realizada por meio das produções e dos relatos dos alunos sobre o tema estudado.

\section{DISCUSSÕES DAS ATIVIDADES}

Qualquer prática educativa se fundamenta numa concepção de ser humano, numa visão de mundo e num modo de pensar os processos de humanização e formação do ser humano (CALDART, 2010). É isso que se busca com atividades que levem o aluno a pensar sobre tudo o que acontece em sua volta com vontade de mudar sua realidade. Molina \& Sá (2012, p.330) argumentam que

Uma das principais características exitosas desta estratégia de vinculação dos processos e ensino-aprendizagem com a realidade social, e com as condições de reprodução material dos educandos que frequentam a escola do campo, refere-se à construção de estratégias pedagógicas que sejam capazes de superar os limites da sala de aula, construindo espaços de aprendizagem que extrapolem esses limite, e que permitam a apreensão das contradições do lado de fora da sala.

É diante dessa perspectiva que o trabalho com informações estatísticas referentes à cultura de tabacos foi realizado em sala de aula. 
Para Santos e Mortimer (2000) as atividades em sala de aula são recomendadas para que se discutam e reflitam problemas sócio-científicos associados à realidade e por meio desses conceitos formarem atitudes e valores com a finalidade de preparar o aluno para questionar, participar e construir coletivamente respostas para problemas sociais.

Sendo assim, para promover discussões e ensinar conceitos de estatísticas pertencentes ao currículo de Matemática, tomou-se por base 3 (três) textos que abordavam os temas propostos.

Texto 1 - Análise da conjuntura agropecuária (SEAB/DERAL) - Fumicultura - Safra 2011/12 - (GROXKO, 2011, p. 1-12)

Para introdução da atividade, essa primeira leitura realizada tratava dos aspectos econômicos e relatava informações de dois temas: a produção brasileira de tabacos e a evolução da cultura de tabacos. O texto utilizado é do economista Methodio Groxko da SEAB/DERAL, entidades da agricultura paranaense e trazia em sua estrutura um panorama de informações estatísticas sobre a produção de fumo no Brasil e no Paraná. Abaixo estão relatados alguns aspectos pertinentes e ilustrações dessa atividade.

\section{Tema 1 - Produção brasileira de tabacos}

Esse tema abrange vários aspectos da cultura de tabacos no Brasil voltados a sua evolução. O trabalho com evolução de fenômenos está relacionado na Estatística com dados em que há certo período de tempo em estudo, ou seja, uma série cronoló gica. Nesse caso o período utilizado foi de 10 (dez) anos de 2000 -2010. Para tanto, utilizou-se os conceitos de tabelas simples e tabelas de dupla entrada para representar os dados em questão, pois, ambas possuem características relativas para representar dados de séries cronológicas. A tabela simples "é aquela que apresenta dados ou informações relativas a uma única variável” (GONZÁLES, 2008, p.13) e tabela de dupla entrada "é a que apresenta, por sua vez, dados ou informações relativas a mais de uma variável" (GONZÁLES, 2008, p.13). Abaixo estão representadas exemplos dessas construções.

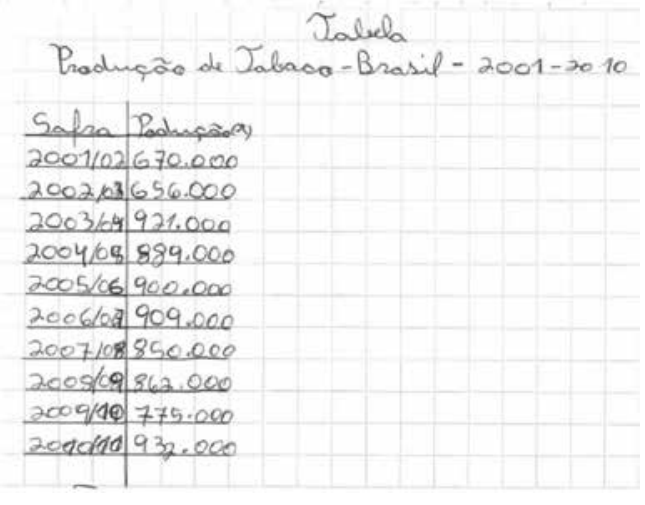

Figura 1 - Tabela simples com informações sobre a cultura de tabacos. Fonte: Arquivos da Autora 


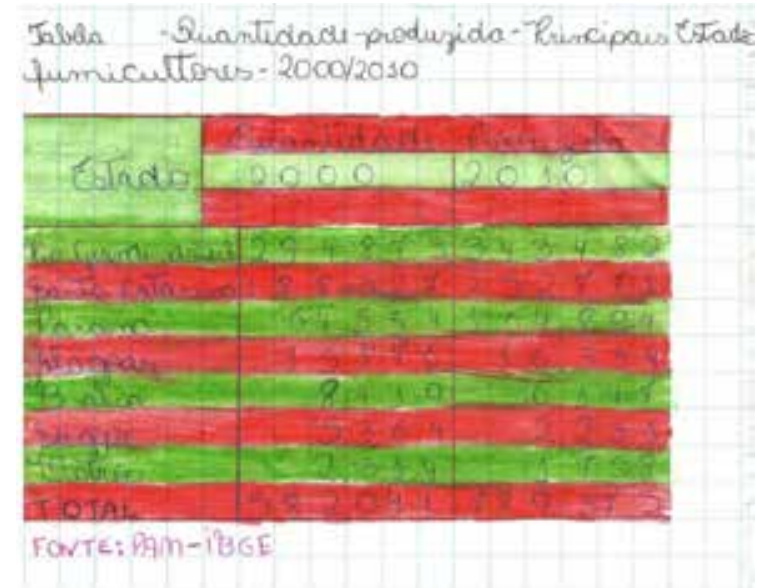

Figura 2 - Tabela de dupla entrada com informações sobre a cultura de tabacos. Fonte: Arquivos da Autora

Com as reflexões sobre o tema e as produções dos alunos se traçou um panorama histórico da economia agrícola da cultura de tabacos no Brasil. Ao trabalhar com essas informações estatísticas, além da leitura e do conhecimento de informações pertinentes a realidade desses alunos, houve também a aprendizagem de conceitos de tabelas estatísticas de acordo com suas normas técnicas e também a sua utilização para representar dados de fenômenos de séries cronológicas, o que vai ao encontro das argumentações de Santana (2011, p.22):

[...] é pertinente e relevante buscarmos promover na Educação Básica um processo de ensino e aprendizagem que possa levar em conta a natureza do conhecimento estatístico, as implicações éticas de seu uso, e não nos limitarmos a um trabalho procedimental e mecanicista.

E ainda nessa perspectiva, podemos destacar no contexto dos estudos CTS que:

[...] é importante que os estudos CTS sejam mesclados às abordagens técnicas que prevalecem no ensino de Matemática, para que a inclusão das preocupações com os aspectos sociais da análise da ciência e da tecnologia desencadeiem uma quebra da postura tradicional de transmissão de conteúdos, bem como uma motivação maior para a aprendizagem, desenvolvendo nos alunos a capacidade crítica para analisar os aspectos sociais e ambientais derivados das novas tecnologias ou do uso de ferramentas matemáticas e tecnológicas em aplicações científicas (CURY \& BAZZO, 2001, p.42).

Logo, os conteúdos matemáticos trazem por meio de seus conceitos uma maneira de mostrar dados que fazem parte dessa realidade escolar.

\section{Tema 2: Evolução da cultura da tabacos}

O tema 2 também é retirado do texto 1 . O conceito estatístico utilizado nesse contexto é gráfico de linhas. Esse tipo de gráfico é frequentemente utilizado para representar séries cronológicas (GONZÁLES, 2008).

Na figura 3 estão representadas as flutuações da produção de cultura de tabacos entre os anos de 2001 e 2010. E esse intervalo de tempo faz parte da época de vida desses alunos do $6^{\circ}$ ano. O que caracteriza a atividade como um trabalho com cultura e identidade. 
Figura 3 - Gráfico de linhas com informações sobre a cultura de tabacos. Fonte: Arquivos da Autora

No enfoque CTS, podemos destacar que o trabalho com informações que envolvem evolução nem sempre pode ser uma evolução positiva, pois nesse caso, não estão considerados os aspectos humanos e ambientais, apenas o econômico. "Assim, é preciso tomar muito cuidado ao recebermos tais informações, pois muitos dados importantes podem estar escondidos por detrás dos números, das probabilidades, enfim, de valores que nos parecem inocentes”. (PINHEIRO \& BAZZO, 2004, p. 2).

A evolução da cultura de tabacos aconteceu, mas os impactos na saúde dos fumicultores e no meio ambiente não foram contabilizados nessa evolução.

Texto 2 - Agrotóxicos e o risco à saúde entre fumicultores. (ALMEIDA et al., 2011, p. 57-63 )

Esse segundo texto foi utilizado por se tratar de uma pesquisa realizada com fumicultores de um município vizinho ao que os alunos residem. O texto traz informações sobre os sintomas causados por intoxicação crônica de agrotóxicos em fumicultores. Os alunos ao coletarem essas informações do fragmento desse artigo, mostraram-se perplexos, pois, relataram situações semelhantes que acontecem com seus pais após o manejo de agrotóxicos nas plantações de fumo. Como nas falas relatadas abaixo:

“O meu pai chega todos os dias do "fumá” com dor de cabeça” (ALUNO 1)

“Tinha que ter um outro jeito de plantar fumo que não precisasse de veneno para pragas” (ALUNO 2)

Esses depoimentos são ricos de significados e pontuam a vivência cultural desses estudantes, mesmo em meio ao perigo, acreditam que a melhor saída não é acabar com a cultura de tabacos e sim melhorar as formas de plantio e a qualidade de vida.

Tema 3: Impacto da cultura de tabacos na saúde dos fumicultores

O conteúdo matemático utilizado para tratar as informações sobre este tema foi o gráfico de barras simples. Esse tipo de gráfico é ideal pra representar dados qualitativos. Na figura 5, está ilustrada a representação dos dados retirados do texto. 


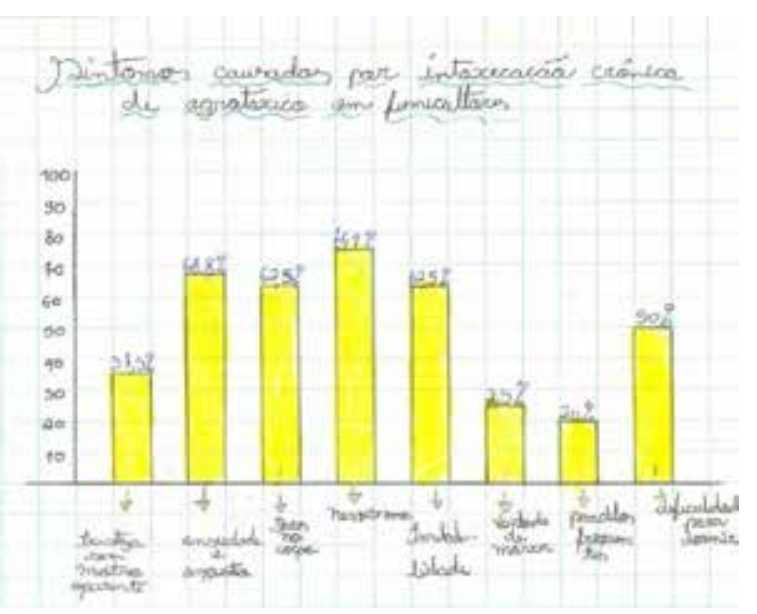

Figura 5 - Gráfico de barras simples com informações sobre a cultura de tabacos. Fonte: Arquivos da Autora

Nesse estudo partiu-se de um tema social próximo da realidade dos alunos para trabalhar o conteúdo estatístico. O estudo do conteúdo científico definido em função do tema social e da tecnologia introduzida é o ensino de estatística.

Com o ensino de estatística pontuamos esse aspecto científico cultural, ao analisar as os impactos na saúde dos fumicultores. Ensinar as técnicas de construção de um tipo de gráfico, com dados de um tema que está relacionado com a realidade desses alunos torna o ensino mais motivador. E o compromisso principal do trabalho com esse tema é formar alunos críticos capazes de questionar a realidade que os cerca.

Texto 3 - Impacto da restrição ao uso de aditivos em derivados de tabaco nos principais municípios fumicultores do Paraná. (ZANCHET \& LIMA, 2012, p.58-71)

Este terceiro texto foi utilizado por trazer em sua estrutura dados referentes à cultura de tabacos da região em que vivem os estudantes dessa escola do campo. Além disso, apresenta dados referentes ao município em que eles moram, o que mostra uma característica da cultura e da identidade deles. Na sequência apresenta-se um breve relato dessa atividade e sua articulação com a realidade social desses estudantes.

\section{Tema 4 - Produção Regional de tabacos}

A Educação do Campo propõe que a realidade do aluno seja trabalhada em consonância com a cultura e a identidade desses educando. Pois, a identidade e cultura dos povos do campo devem ser valorizadas para o reconhecimento desses sujeitos que possuem laços culturais e valores relacionados à vida na terra (PARANÁ, 2007). Diante disso, o segundo texto utilizado para reflexão relaciona as informações sobre a pauta produtiva de tabacos com as principais regiões fumicultoras ligadas ao fato da proibição do uso de aditivos na cultura, onde cita o municio que eles vivem e também municípios vizinhos.

O texto apresentava uma tabela estatística, para tanto, com as informações dessa tabela foi possível realizar a contextualização do conceito de gráfico de barras múltiplas. Esse tipo de gráfico "é geralmente empregado quando queremos representar, simultaneamente, dois ou mais fenômenos estudados com o propósito de comparação" (CRESPO, 2009, p.35). Com essa atividade além da aprendizagem de outro tipo de gráfico, também foi possível proporcionar ao estudante a habilidade de realizar a transnumeração de informações estatísticas, que ocorre cada vez que mudamos nossa maneira de observar os dados e isso nos conduz a atribuir novos significados (WILD \& PFANNKUCH, 1999). A figura abaixo ilustra transnumeração realizada pelos estudantes. 


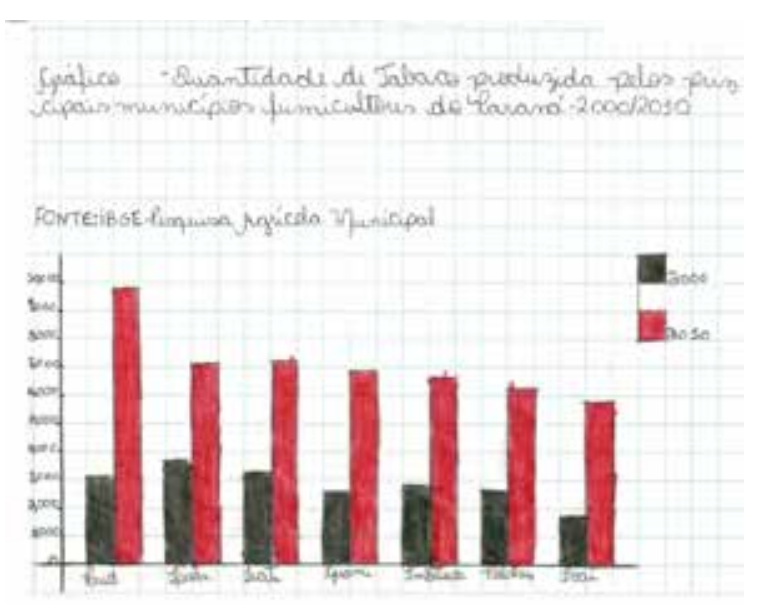

Figura 4 - Gráfico de barras múltiplas com informações sobre a cultura de tabacos. Fonte: Arquivos da Autora

Com essa representação é possível observar a comparação da cultura agrícola que os cerca e também entender o campo como um modo de vida social e que suas contribuições afirmam a identidade dos povos do campo, valorizando "o seu trabalho, a sua história, o seu jeito de ser, os seus conhecimentos, a sua relação com a natureza e como ser da natureza” (PARANÁ, 2007, p.26).

Na perspectiva CTS destaca-se que a "interação entre ciência, tecnologia e sociedade propiciaria o desenvolvimento de valores e idéias por meio de estudos de temas locais, políticas públicas e temas globais" (SANTOS \& MORTIMER, 2000, p. 137). E essa foi uma interação constatada no trabalho com essas informações e com essa cultura relacionada ao cotidiano desses estudantes.

\section{APRESENTAÇÃO DAS ATIVIDADES PARA A COMUNIDADE}

Após a realização das reflexões e das construções estatísticas a turma foi dividida em grupos que apresentaram em banners para a comunidade, em uma feira de conhecimento realizada anualmente por essa escola do campo. A figura 5 é uma imagem de um dos banners utilizado nas apresentações.

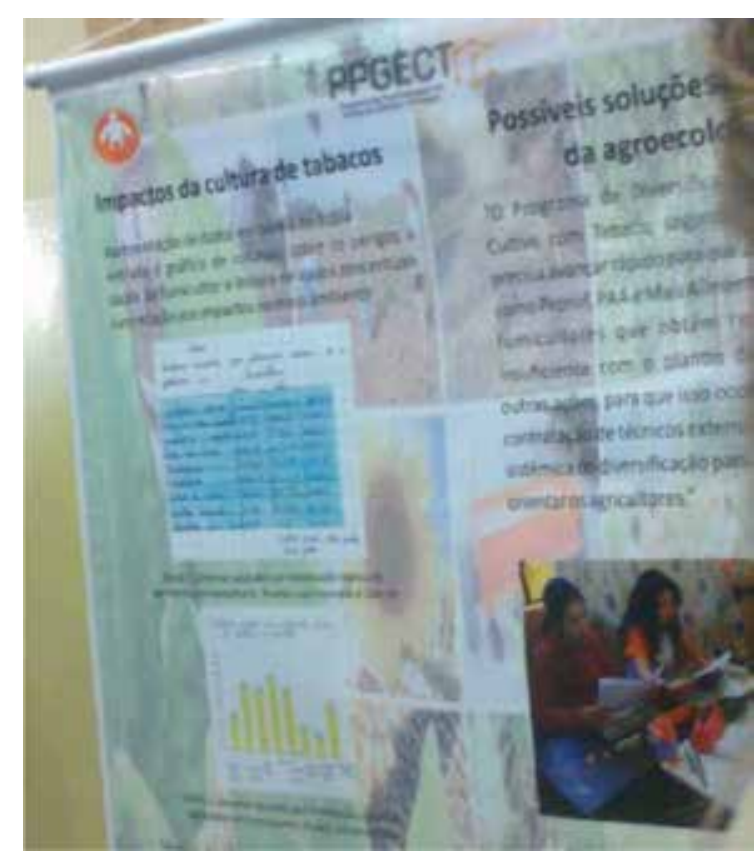

Figura 5 - Banner com as informações sobre a cultura de tabacos trabalhadas pelos alunos. Fonte: Arquivos da Autora 
Ao apresentar para a comunidade suas produções os alunos se motivaram também em mostrar que a aprendizagem em sala de aula teve significados.

É nesta perspectiva que os estudos CTS foram realizados contemplando as seguintes etapas:

- Introdução de uma temática social: a cultura de tabacos e sua implicação na sociedade;

- Tecnologia relacionada ao tema: a evolução tecnológica da cultura de tabacos e seu crescimento perante as exportações internacionais;

- Estudo dos conteúdos: tratamento de dados estatísticos, organização de dados em tabelas e gráficos;

- Discussão da problemática social: mesmo sendo uma das culturas com boa rentabilidade econômica apresenta riscos à saúde aos trabalhadores.

Sendo assim, fica evidenciado que a identificação dos aspectos organizacionais e culturais da tecnologia mostra que ela é dependente de sistemas sócio-políticos, valores e ideologias da cultura em que se insere (SANTOS \& MORTIMER, 2000).

A cultura de tabacos é uma cultura dependente da política social onde a tecnologia agronômica se faz presente e a qualidade de vida pode estar em decadência.

\section{CONSIDERAÇÕES FINAIS}

As práticas educativas de sala de aula podem trazer significados para os estudantes quando forem trabalhadas dentro do contexto social em que este está inserido.

Os estudos CTS, nessa linha, apresentam suporte para o trabalho com a realidade. Ler, discutir, refletir e usar essas leituras para aprender conceitos relativos a um conteúdo específico da matemática é articular conhecimento escolar com o conhecimento social. 
As atividades realizadas contribuíram para a aprendizagem de técnicas de organização de dados estatísticos e foram propostas com o objetivo de formar alunos reflexivos capazes de discutir a realidade que os cerca.

\section{REFERÊNCIAS}

ALBUQUERQUE, C. Método soma-capacitação de agricultores, educação sanitária e ambiental. Edit. Bandeirante, Goiânia, 2000.

ALMEIDA, E. A. de; ZIMMERMAN, M.H; GONÇALVES, C. dos S; GRDEN, C. R. B; MACIEL, M.A.S; BAIL, L; ITO, C.A S. Agrotóxicos e o risco à saúde entre fumicultores. Publ. UEPG Ci. Biol. Saúde, Ponta Grossa, v.17, n.2, p. 57-63, jul./dez. 2011. Disponível em http://www.revistas2.uepg.br/index.php/biologica. Acesso em agosto de 2012.

ANELE, A.C. O enfoque CTS em sala de aula: Uma abordagem diferenciada utilizando a Unidade de Aprendizagem em Educação Química. 2007. 109 f. Dissertação (Mestrado em Educação em Ciências e Matemática) - Faculdade de Física, Pontifícia Universidade Católica do Rio Grande do Sul, Porto Alegre.

BATANERO, C. Controversies around the role of statistical tests in experimental research. Mathematical Thinking and Learning 2 (1-2), 2000.

BAZZO, W. A.(1998): Ciência, Tecnologia e Sociedade: e o contexto da educação tecnológica. Florianópolis: Editora da UFSC, 1998.

BAZZO, W. A. A pertinência de abordagens CTS na educação tecnológica. Revista Iberoamericana de Educación, n. 28, p. 83-99, jan./abr. 2002.

BRASIL. AGÊNCIA NACIONAL DE VIGILÂNCIA SANITÁRIA (ANVISA). Brasília, DF, 2008. Disponível em: www.anvisa.gov.br. Acesso em10 de julho de 2012.

CALDART, R. S. A educação do campo e a perspectiva de transformação da forma escola. In: MUNARIN, A . et al. (org). Educação do campo: reflexões e perspectivas. Florianópolis: Insuflar, 2010.

CRESPO, A. A. Estatística fácil. 19a. Ed. São Paulo: Saraiva, 2009.

CURY, H. N \& BAZZO, W. A. Formação crítica em Matemática: uma questão curricular? Bolema, Rio Claro, v. 14, n. 16, p. 29-47, 2001.

FRANCO, M. A. S. Pedagogia da Pesquisa-Ação. Educação e Pesquisa, São Paulo, v. 31, n. 3, p. 483-502, set./ dez. 2005. Disponível em: http://www.scielo.br/pdf/ep/v31n3/a11v31n3.pdf , acesso em 03 de junho de 2013.

GLANTZ, S; SIADE, J.; BERO, L; HANAUER, P; BARNERS, D. The cigarette papers. Berkeley and Los Angeles: University of California Press, 1996.

GONZÁLES, N. Estatística Básica. Rio de Janeiro: Editora Ciência Moderna Ltda, 2008.

GROXKO, M. Análise Conjuntural da Fumicultura. SEAB/DERAL. Prognóstico agropecuário.2011. Disponível em: http://www.agricultura.pr.gov.br/arquivos/File/deral/Prognosticos/fumicultura_2011_12.pdf. Acesso em: 20 ago. 2012.

LEFF, H. Saber ambiental: sustentabilidade, racionalidade, complexidade, poder. Tradução de Lúcia Mathilde Endilich Orth. Petrópolis: Vozes, 2004.

LOPES, C. E. O estudo da estatística e da probabilidade na educação básica e a formação dos professores. Artigo. Campinas, São Paulo, 2008. 
MOLINA, M. C \& SÁ, L. M. Escola do campo. IN: CALDART, R. S. et al (org). Dicionário da Educação do Campo. Rio de Janeiro, São Paulo: Escola Politécnica de Saúde Joaquim Venâncio, Expressão Popular, 2012.

PARANÁ. Secretaria de Estado da Educação do Paraná. Diretrizes curriculares da Educação do campo. Paraná: SEED, 2007.

PINHEIRO, S. \& LUZ, D. Ladrões de Natureza: uma reflexão sobre a biotecnologia e o futuro do planeta. Porto Alegre: Fundação Juquira-Candiru, 1998.

PINHEIRO, N. A. M. \& BAZZO, W. A. Uma Experiência Matemática Sob o Enfoque CTS: Subsídios para discussões. In: Perspectiva (Erexim), Erechim, v. 28, n. 103, p. 33-49, 2004.

SANTANA, M. de S. A educação estatística com base num ciclo investigativo: um estudo do desenvolvimento do letramento estatístico de estudantes de uma turma do $3^{\circ}$ ano do ensino médio . 2011. Dissertação (Mestrado em Educação Matemática) - Universidade Federal de Ouro Preto

SANTOS, W. L. P. dos \& MORTIMER, E. F. Uma Análise de Pressupostos Teóricos da Abordagem C-T-S (Ciência-Tecnologia-Sociedade) no Contexto da Educação Brasileira. Ensaio - pesquisa em educação em ciências, v. 2, n. 2, p.133-162, 2000.

SILVEIRA, R. M. C. F. Inovação tecnológica na visão dos gestores e empreendedores de Incubadoras de Empresas de Base Tecnológica do Paraná (IEBT-PR): desafios e perspectivas para a educação tecnológica. 2007. 274 f. Tese (Doutorado) - Universidade Federal de Santa Catarina, Florianópolis, 2007.

VON LINSINGEN, I. Perspectiva educacional CTS: aspectos de um campo em consolidação na América Latina. Revista Ciência \& Ensino, v.1, número especial, p. 1-19, nov. 2007.

WILD, C. \& PFANNKUCH, M. Statistical thinking in empirical enquiry. International Statistical Review, n.67, p. 223-65, 1999. Disponível em: <http://www.stat.aucland.ac.nz/ iase/publications/isr/99.wild. pfannkuch. pdf $>$. Acesso em: agosto de 2012.

ZANCHET, M. S. \& LIMA, F. R. S. de. Impacto da restrição ao uso de aditivos em derivados de tabaco nos principais municípios fumicultores do Paraná. Caderno Ipardes. Curitiba, PR. V.2, n.1, p.58-71, jan/jun.2012

ZANON, J.S \& WIZNIEWSKY, C.R.F. Desenvolvimento Rural sustentável: resgatando saberes na escola municipal de ensino funadamental Bernardino Fernandes, Distrito Pains, Santa Maria - RS. IN :VI ENCONTRO DE PESQUISA. Presidente Prudente (SP), 2011. Anais...disponível em: http://www4.fct.unesp.br/encontros/ engrup/Trabalhos/TEXTOS-POSTERS-PUBLICACAO/GPET_Joao_Silvano_DRS_Escolas_Municipais_ Santa_Maria.pdf. Acesso em 30 de maio de 2013. 